\title{
Intermolecular Effects of Fluorocarbons on MC800 Asphaltene and their Characterization
}

\author{
H.E. Kirimli*, H. Ovalioglu and C. Akay \\ Uludag University, Faculty of Arts and Sciences, Department of Physics, 16059, Bursa, Turkey
}

\begin{abstract}
Nuclear magnetic resonance and dynamic nuclear polarization experiments were performed to study suspensions of asphaltene in the fluorocarbons at a low magnetic field of $1.53 \mathrm{mT}$, at room temperature. The asphaltene was extracted from MC800 liquid asphalt, which was of Heavy Iran origin. The elemental composition and the surface morphology of the asphaltene were determined by scanning electron microscopy and energy dispersive X-ray analysis. Intermolecular spin-spin interactions occur between nuclear spins of hydrogen in the solvent medium and the free electron spins in the asphaltene micelles. The electron paramagnetic resonance spectrum of the asphaltene was obtained and the saturation experiments were applied to the samples prepared in vacuum. The main ${ }^{1} \mathrm{H} D N P$ parameters were listed. Chemical composition and molecular structure of the asphaltene were analyzed. Scanning electron microscope images have shown that asphaltenes are constituted of agglomerated particles and smooth surfaces. Energy dispersive X-ray analysis has shown that the most abundant element is carbon.
\end{abstract}

DOI: 10.12693/APhysPolA.131.336

PACS/topics: $76.70 . \mathrm{Fz}, 83.80 . \mathrm{Hj}, 68.37 . \mathrm{Hk}, 61.72 . \mathrm{Ff}$

\section{Introduction}

Asphaltenes, which are a part of petroleum, are regarded to be polar species, have larger aromatic structures and are containing alkyl chains, heteroatoms (such as $\mathrm{O}$, $\mathrm{S}$ and $\mathrm{N}$ ) and some metals [1]. Asphaltenes have unpaired electrons, which are observed using the electron spin resonance (ESR) experiments in crude petroleum [2].

Dynamic nuclear polarization (DNP) utilizes the liquid state interaction between nuclei and unpaired electron spins to generate an increased nuclear polarization upon the saturation of ESR transitions of the radicals. The maximum DNP enhancement of the NMR signal is given by the electron-to-nuclear gyromagnetic ratios $\gamma_{\mathrm{e}} / \gamma_{\mathrm{n}}$. This ratio for proton spin is 660 . In this study, the nuclear spins are the proton spins, which are the ${ }^{1} \mathrm{H}$ nuclei of the solvent medium and the electron spins belong to the free electrons on the asphaltene micelles. The unpaired electrons were delocalized on the incomplete carbon bonds of the condensed aromatic structure of the asphaltene particles [3]. By DNP method we have revealed dipolar interaction between hydrogen atoms of the solvent and unpaired electrons of colloidal asphaltene. Electron spin saturation and DNP enhancements have been obtained and DNP parameters determined in low magnetic field. Chemical composition and morphology of MC800 asphaltene were investigated using scanning electron microscopy (SEM) and energy-dispersive X-ray (EDX) analysis.

\section{Materials and equipment}

The equipment and technical details of the double resonance spectrometer used in this work have been extensively described in prior publications [4-6]. Further

\footnotetext{
*corresponding author; e-mail: hengin@uludag.edu.tr
}

information on the theory and applications of DNP for liquid samples is available in literature [6-9]. DNP experiments were performed at $1.53 \mathrm{mT}$ with a resonance frequency of $61.2 \mathrm{kHz}$ for the ${ }^{1} \mathrm{H}$ nuclei and $42.6 \mathrm{MHz}$ for the electrons. The asphaltene was extracted from liquid asphalt MC800. The asphalt was taken from the Tupras, Refinery in Kirikkale, Turkey. The suspensions were prepared in three different asphaltene concentrations. The solvents were purchased from Aldrich Chemical Co. (USA) (Table I). The samples in Pyrex tubes were degassed and sealed.

\section{TABLE I}

Basic constants and NMR sensitivity of selected solvents. (1) Fluorobenzene $\left(\mathrm{C}_{6} \mathrm{H}_{5} \mathrm{~F}\right),(2)$ o-Difluorobenzene $\left(\mathrm{C}_{6} \mathrm{H}_{4} \mathrm{~F}_{2}\right)$, (3) m-Difluorobenzene $\left(\mathrm{C}_{6} \mathrm{H}_{4} \mathrm{~F}_{2}\right)$, (4) p-Difluorobenzene $\left(\mathrm{C}_{6} \mathrm{H}_{4} \mathrm{~F}_{2}\right)$.

\begin{tabular}{c|c|c|c|c|c}
\hline \hline $\begin{array}{c}\text { Sol- } \\
\text { vent }\end{array}$ & $\begin{array}{c}\text { Molecular } \\
\text { weight } \\
{\left[\mathrm{g} \mathrm{mol}^{-1}\right]}\end{array}$ & $\begin{array}{c}\text { Boiling } \\
\text { point } \\
{\left[{ }^{\circ} \mathrm{C}\right]}\end{array}$ & $\begin{array}{c}\text { Melting } \\
\text { point } \\
{\left[{ }^{\circ} \mathrm{C}\right]}\end{array}$ & $\begin{array}{c}\text { Density } \\
{\left[\mathrm{g} \mathrm{cm}^{-3}\right]}\end{array}$ & $\begin{array}{c}\text { NMR } \\
\text { sensitivity } \\
\left(\times 10^{22} \text { spin } / \mathrm{cm}^{3}\right)\end{array}$ \\
\hline$(1)$ & 96.10 & 85 & -42 & 1.024 & 0.64 \\
$(2)$ & 114.09 & 92 & -34 & 1.158 & 1.22 \\
$(3)$ & 114.09 & 83 & -59 & 1.163 & 1.23 \\
$(4)$ & 114.09 & $88-89$ & -13 & 1.170 & 1.24
\end{tabular}

The surface morphology and chemical composition analysis of the asphaltene were examined using a LEO 1430-VP SEM (LEO Electron Microscopy Ltd., Cambridge, UK) operated at $20 \mathrm{kV}$, with energy dispersive X-ray analysis capabilities. EDX analysis was used to identify the elemental composition of localized areas on sample particle surfaces.

\section{Results and discussion \\ 3.1. EPR spectra}

It is essential to obtain the EPR spectrum of free radical to measure the DNP parameters in a given magnetic 
field. ESR spectrum should not be affected by the solvents [4]. Figure 1 shows the EPR spectrum of the asphaltene/fluorobenzene solvent medium for three different concentrations, at room temperature. The spectrum was considered as a single Gaussian, which has been formed by the superposition of several Lorentzians. The measured values of the spectrum with the biggest concentration are best fit with a Gaussian, given by the following equation:

$$
-\frac{\left(P_{z}-P_{0}\right)}{P_{0}}=0.61 \exp \left[-\frac{\left(v_{s}-42.58\right)^{2}}{37.67}\right] .
$$

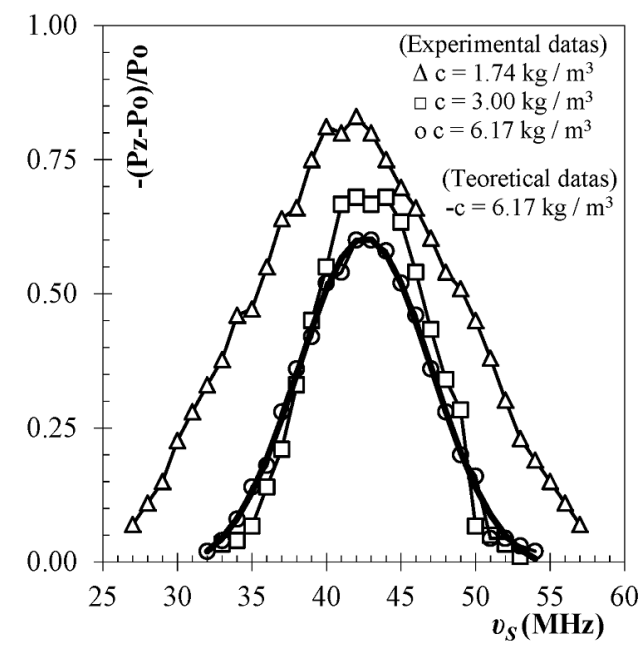

Fig. 1. EPR spectrum of the asphaltene/fluorobenzene solvent medium for three different concentrations. The fitted curve for $6.17 \mathrm{~kg} / \mathrm{m}^{3}$ radical concentration is a single Gaussian with $42.58 \mathrm{MHz}$ peak frequency and 4.34 MHz standard deviation. The thick line represents the fitted curve.

\subsection{DNP parameters}

The NMR enhancement factor is defined as [10]

$$
A=\frac{P_{z}-P_{0}}{P_{0}}=-\rho f s\left|\frac{\gamma_{S}}{\gamma_{I}}\right|,
$$

where $P_{0}$ and $P_{z}$ are the NMR signal amplitudes without and with ESR saturation power, $\gamma_{S}$ and $\gamma_{I}$ are electronic and nuclear gyromagnetic ratios, respectively, $f$ is the leakage factor for the nuclear relaxation, $s$ is the saturation factor, which is equal to 1 for the complete EPR saturation, $\rho$ is the nuclear-electron coupling parameter, which is a measure of the nuclear-electron interaction. All samples were saturated at $42.6 \mathrm{MHz}$. The variation of $-\left[\left(P_{z}-P_{0}\right) / P_{0}\right]^{-1}$ versus $V_{\mathrm{eff}}^{-2}$ for asphaltene/odifluorobenzene sample at three different concentrations is given in Fig. 2a. $A^{-1}$ is equal to $A_{\text {end }}^{-1}$ for the maximum obtainable EPR power. If the saturation condition is fulfilled, the reciprocal of the enhancement factor is extrapolated for the infinite ESR power. $A_{\infty}$ is the enhancement factor at complete EPR saturation and it is given as

$$
A_{\infty}=-\rho f\left|\frac{\gamma_{S}}{\gamma_{I}}\right| .
$$

Since $A_{\infty}^{-1}$ values are proportional to the reciprocal of the radical concentration $c^{-1}$, the ultimate enhancement factor $U_{\infty}$ can be easily found (Fig. 2b). The values of $\rho$ can be calculated after determination of $U_{\infty}$.

$$
\rho=-\frac{U_{\infty}}{658} .
$$
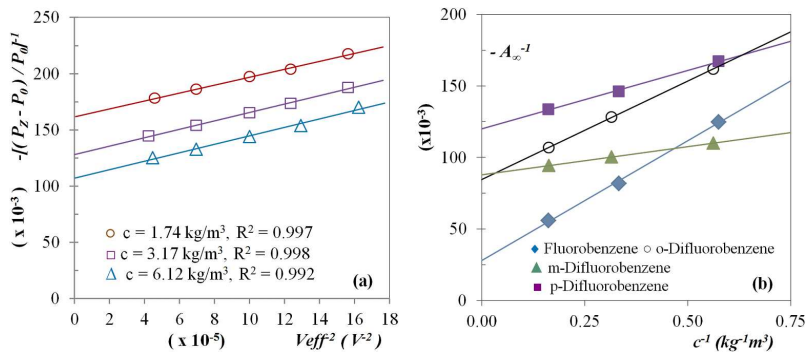

Fig. 2. (a) Variation of $-\left[\left(P_{z}-P_{0}\right) / P_{0}\right]^{-1}$ versus $V_{\text {eff }}^{-2}$ for asphaltene/o-difluorobenzene sample at three different concentrations. $A_{\infty}^{-1}$ values correspond to the intersection points of the $V_{\text {eff }}^{-2}=0$ line and the extrapolated best-fit line. The $R^{2}$ values show the degree of agreement between the linear fit function and the experimental points. (b) Examples of the determination of ultimate enhancement factors. $U_{\infty}^{-1}$ values correspond to the intersection points of the $c^{-1}=0$ line and the extrapolated best-fit lines.

$f$ value for each concentration was calculated from the ratio of $A_{\infty} / U_{\infty}$, and the $s$ value was found as the ratio of $A_{\text {end }}$ to $A_{\infty}$. The dynamic nuclear polarization parameters, $A_{\infty}, U_{\infty}, \rho, f$ and $s$ are given in Table II. Experimental saturations are between 78 and $96 \%$. Value of $A_{\infty}$ varies between -17.8 and -3.0 . It increases for all samples, as sample concentration increases. All $A_{\infty}$ and $U_{\infty}$ values are negative because $P_{z}$ and $P_{0}$ NMR signals are opposite to each other. This situation also causes nuclear coupling parameter $\rho$ to be positive for all solvent media. $\rho$ value of asphaltene/fluorobenzene is 0.055 , which is the biggest $\rho$ value among all samples. This means that fluorobenzene is the most effective solvent to disperse and suspend the asphaltene among any of tested solvents. $\rho$ values of o-difluorobenzene, mdifluorobenzene and p-difluorobenzene change according to the electronic structures and the chemical environment of ${ }^{1} \mathrm{H}$ nuclei of these three isomers.

\subsection{Chemical composition and morphology of asphaltene}

Figure 3a shows the SEM images for asphaltene obtained from liquid asphaltene and Fig. 3b shows EDX spectra of asphaltene particle. In the morphology of asphaltene a smooth surface, with variable particles of irregular shapes deposited on it, were observed. In Fig. 3a focus was made on checking the amorphous white particles of different sizes. As seen in Table III, carbon element is most abundant, in accordance with Trejo [11] studies. Carbon is followed by phosphorus and sulfur. Trace metal amounts $(\mathrm{Ni}, \mathrm{V})$ are found. 
TABLE II

DNP parameters for the suspensions of the selected fluorocarbonds with asphaltene.

\begin{tabular}{c|c|c|c|c|c|c}
\hline \hline Solvent medium & $\begin{array}{c}\mathrm{c} \\
{\left[\mathrm{kg} / \mathrm{m}^{3}\right]}\end{array}$ & $A_{\infty}$ & $U_{\infty}$ & $\rho$ & $f$ & $s$ \\
\hline (1)Asphaltene/ & 1.7 & -8.0 & & & 0.220 & 0.841 \\
Fluorobenzene & 3.0 & -12.2 & -36.3 & 0.055 & 0.337 & 0.876 \\
& 6.2 & -17.8 & & & 0.490 & 0.842 \\
\hline \multirow{2}{*}{ (2) Asphaltene/ } & 1.8 & -6.2 & & & 0.523 & 0.908 \\
o-Difluorobenzene & 3.2 & -7.8 & -11.8 & 0.018 & 0.660 & 0.887 \\
& 6.1 & -9.4 & & & 0.791 & 0.855 \\
\hline \multirow{2}{*}{ (3) Asphaltene/ } & 1.8 & -9.1 & & & 0.796 & 0.779 \\
m-Difluorobenzene & 3.1 & -10.0 & -11.4 & 0.017 & 0.872 & 0.843 \\
& 6.3 & -10.6 & & & 0.931 & 0.806 \\
\hline \multirow{2}{*}{ (4) Asphaltene/ } & 1.8 & -6.0 & & & 0.718 & 0.833 \\
p-Difluorobenzene & 3.3 & -6.84 & -8.3 & 0.013 & 0.820 & 0.891 \\
& 6.2 & -7.48 & & & 0.898 & 0.889
\end{tabular}

TABLE III

EDX analysis of the amorphous white particle shown in Fig. 3a.

\begin{tabular}{c|c|c|c|c|c|c}
\hline \hline \multicolumn{7}{c}{ Elemental composition, [wt.\%] } \\
\hline $\mathrm{C}$ & $\mathrm{O}$ & $\mathrm{Si}$ & $\mathrm{P}$ & $\mathrm{S}$ & $\mathrm{V}$ & $\mathrm{Ni}$ \\
\hline 90.98 & 0.14 & 0.20 & 4.62 & 4.03 & 0.01 & 0.01
\end{tabular}
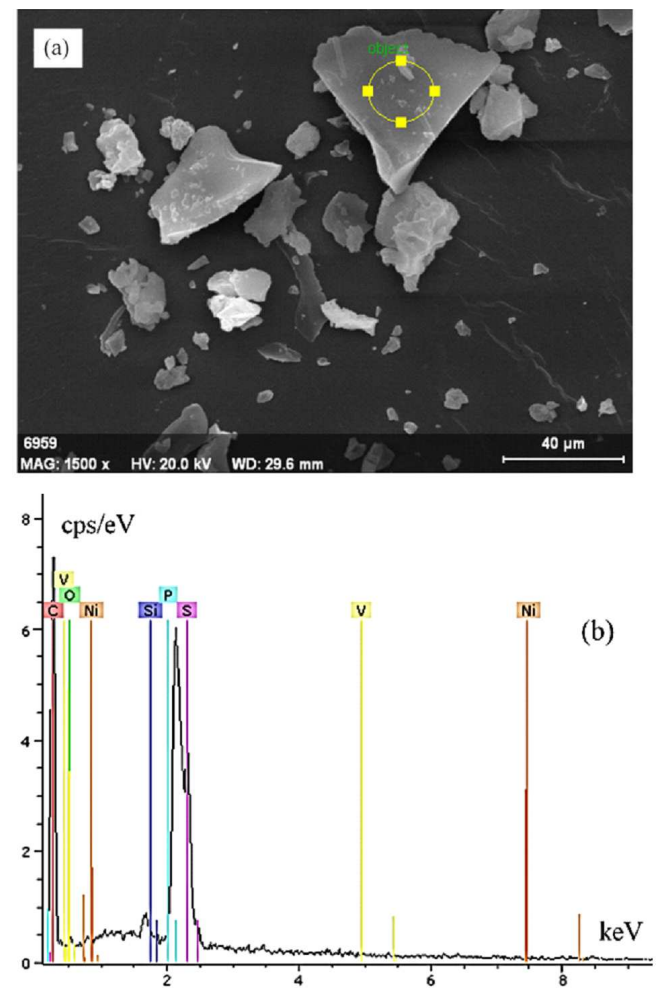

Fig. 3. (a) Microscopic structures of asphaltenes under the SEM at $1500 \times$ and (b) EDX spectra of asphaltene particle.

\section{Conclusions}

DNP experiments were performed to study suspensions of asphaltene in fluorocarbon solvents at $1.53 \mathrm{mT}$. We have observed a single Gaussian which was peaked around $42.6 \mathrm{MHz}$ in the EPR spectra of the asphaltenes. In low magnetic fields, $\rho$ varies from +0.5 (pure dipolar) to -1.0 (pure scalar). In our study, $\rho$, had varied between 0.055 and 0.013 . The interactions occurring between unpaired electrons of the asphaltene micelles and the hydrogen nuclei of the solvent molecules have dipolar character. The obtained DNP parameters demonstrate that asphaltene particles have aggregated under the effect of solvent. Asphaltene particles have such elements as $\mathrm{C}, \mathrm{S}, \mathrm{O}$ and $\mathrm{V}$, according to analysis of chemical composition and elemental mapping. During the analysis of the SEM images, a smooth surface, asphaltene particles with different sizes and asphaltene aggregations were observed. The most abundant element is carbon.

\section{Acknowledgments}

This work was supported by Uludag University, Scientific Research Projects Unit grant no: OUAP-(F)$2012 / 13$. The authors would like to thank Uludag University for financial support.

\section{References}

[1] I.N. Evdokimov, N.Y. Eliseev, B.R. Akhmetov, Fuel 85, 1465 (2006).

[2] H.S. Gutowsky, B.R. Ray, R.L. Rutledge, R.R. Unterberger, J. Chem. Phys. 28, 744 (1958).

[3] C. Griesinger, M. Bennati, H.M. Vieth, C. Luchinat, G. Parigi, P. Hofer, F. Engelke, S.J. Glaser, V. Denysenkov, T.F. Prisner, Prog. Nucl. Mag. Res. Sp. 64, 4 (2012).

[4] A. Yalciner, Comm. Fac. Sci. Univ. Ankara A2, 1 (1978).

[5] C. Akay, A. Yalciner, Z. Naturforsch. 50a, 177 (1995).

[6] H.E. Kirimli, H. Ovalioglu, J. Dispersion Sci. Technol. 35, 255 (2014).

[7] K.H. Hausser, D. Stehlik, Adv. Magn. Reson. 3, 79 (1968).

[8] J. Potenza, Adv. Mol. Relax. Proc. 4, 229 (1972).

[9] W. Müller-Warmuth, K. Meise-Gresch, Adv. Magn. Reson. 11, 1 (1983).

[10] K.D. Kramer, W. Müller-Warmuth, Z. Naturforsch. 19a, 375 (1964)

[11] F.T. Trejo, J. Ancheyta, M.S. Rana, Energy \& Fuels 23, 429 (2009). 